\title{
Cognitive Function After Carotid Endarterectomy: Greater Risk of Decline in Symptomatic Patients With Left Internal Carotid Artery Disease
}

\author{
Mario Bo, MD,* Massimiliano Massaia, MD,* Stefania Speme, MD,* \\ Giorgetta Cappa, MD, ${ }^{*}$ Khenya Strumia, MD, ${ }^{*}$ Paolo Cerrato, MD, + \\ Federico Ponzio, MD, $\ddagger$ and Mario Molaschi, MD*
}

\begin{abstract}
Background: The risk of cognitive decline in patients undergoing carotid endarterectomy (CE) for left internal carotid artery (ICA) (LICA) disease before or after the occurrence of ischemic symptoms has not been fully elucidated. We evaluated whether patients undergoing CE for symptomatic LICA stenosis have greater risk of cognitive decline than patients with asymptomatic LICA disease or right ICA disease. Methods: In a series of patients aged 65 years and older, consecutively undergoing $\mathrm{CE}$ and free from cognitive impairment, cognitive function was evaluated through the age- and education-adjusted Mini Mental State Examination and the Clock Drawing Task at baseline and at the end of the study period (average follow-up: $44.4 \pm 14.3$ months). Results: The analysis included 103 patients (mean age $72.6 \pm 7.0$ years; 73 men), of whom 50 had LICA disease (29 symptomatic). At the end of the study period, Mini Mental State Examination score was reduced in patients with symptomatic LICA disease $(P<.001)$ but not in other patients, whereas the Clock Drawing Task score was reduced in both patients with LICA and right ICA. Patients with symptomatic LICA disease had greater risk of cognitive decline than other individuals, either measured by the Mini Mental State Examination score $(\mathrm{F}=5.18, P=.002)$ or by the Clock Drawing Task score $(\mathrm{F}=$ $9.42, P=.001)$. Conclusions: Patients undergoing CE for symptomatic LICA disease appear to be at increased risk of cognitive decline than other individuals. Further studies are needed to confirm these findings and to evaluate whether LICA endarterectomy before occurrence of cerebrovascular ischemic symptoms may provide additive benefit in the prevention of cognitive decline. Key Words: Elderly-cognitive decline-carotid endarterectomy.
\end{abstract}

(C) 2005 by National Stroke Association

\footnotetext{
From the *Section of Gerontology, Department of Medical and Surgical Disciplines; +Neurology, First Division, Department of Neuroscience; and ‡Vascular Surgery, Department of Cardiovascular Medicine and Surgery, University of Turin, Turin, Italy.

Received June 5, 2005; accepted June 30, 2005.

Address correspondence to Mario Bo, MD, Section of Gerontology, Department of Medical and Surgical Disciplines, Ospedale San Giovanni Battista-Molinette, C.so Bramante 88, 10126 Torino, Italia

E-mail: mario.bo@unito.it.

$1052-3057 / \$$ - see front matter

(c) 2005 by National Stroke Association

doi:10.1016/j.jstrokecerebrovasdis.2005.06.003
}

Vascular dementia $(\mathrm{VaD})$ and Alzheimer's dementia (AD) are the most common causes of dementia among elderly people. ${ }^{1}$ Recent basic and clinical investigations have provided evidence that $\mathrm{AD}$ and $\mathrm{VaD}$, traditionally considered distinct pathophysiologic entities, share several common clinical and neuropathologic features, ${ }^{2}$ and some common cerebrovascular risk factors (e.g., hypertension, diabetes, and atrial fibrillation). ${ }^{2,3}$ Ischemic cerebrovascular lesions may coexist with typical neurodegenerative alterations,,$^{2-4}$ and the presence of ischemic lesions enhance the cognitive deficit in patients with AD pathology. ${ }^{5-7}$ Moreover, there is consistent evidence that patients who had ischemic cerebrovascular events have a 
greater risk of developing dementia. ${ }^{8-12}$ In older individuals, mixed dementia is probably more prevalent than typical neurodegenerative dementia or $\mathrm{VaD}^{2,3}$ The definition of vascular cognitive impairment has been proposed to identify all forms of mild to severe cognitive impairment associated with and presumed to be caused by cerebrovascular disease. ${ }^{13,14}$

Stenosis of extracranial carotid artery is a well-defined cause of ischemic cerebrovascular accidents. Although some studies have suggested that hemodynamic asymptomatic carotid stenosis may be associated with a substantial risk of cognitive impairment, ${ }^{15,16}$ the association between asymptomatic carotid atherosclerosis and cognitive impairment is far from definite. ${ }^{17,18}$ In a recent study, it was shown that cognitive impairment and decline are associated with asymptomatic high-grade stenosis of the left internal carotid artery (ICA) (LICA), and that this association is not a result of underlying risk factors or atherosclerosis in general. ${ }^{16}$ This distinction is important because a direct intervention on carotid stenosis, such as endoarterectomy, might prevent cognitive decline. Carotid endarterectomy (CE) has been shown to be effective in reducing the risk of ischemic events in well-defined groups of patients with significant stenosis of the extracranial carotid artery, ${ }^{19}$ but its effects on cognitive function are less well defined. ${ }^{20-26}$ Specifically, one major question that has not been fully elucidated in previous studies is whether patients undergoing CE for symptomatic LICA disease have greater risk of cognitive decline than do asymptomatic patients. In that case, there might be a potential additive benefit in the prevention of cognitive decline from CE on LICA before occurrence of cerebrovascular ischemic symptoms. On this background, we hypothesized that patients who undergo CE for symptomatic LICA stenosis may have greater risk of cognitive decline than patients who receive $\mathrm{CE}$ for asymptomatic LICA stenosis or for right ICA (RICA) disease, either symptomatic or asymptomatic. Therefore, in this study we aimed to evaluate whether older patients undergoing CE for symptomatic LICA disease experience greater cognitive decline than those receiving $\mathrm{CE}$ for asymptomatic LICA stenosis or for RICA disease.

\section{Methods}

Patients 65 years and older eligible to receive CE and who were free from dementia or cognitive impairment were eligible for the study. Patients were enrolled at the day hospital of the vascular surgery department of a university teaching hospital in Turin, Italy. Consecutive patients admitted to the vascular surgery day hospital with a diagnosis of stenosis of the extracranial segment of the ICA and who were scheduled for CE during the period January through September 1999 were requested to participate in the study. The following variables were considered: demographics (age, sex); conventional cardiovascular risk factors (cigarette smoking, diabetes, hypertension, hypercholesterolemia); presence of peripheral obstructive arterial disease (ankle-arm index $<0.90$ ); and history of coronary heart disease (previous myocardial infarction, stable or unstable angina, percutaneous or surgical coronary revascularization). Hypertension was defined as a blood pressure of 160/90 mm Hg or greater, or the use of antihypertensive medications. Diabetes mellitus was considered present if a random glucose reading was $140 \mathrm{mg} / \mathrm{dL}$ or greater, a 12-hour fasting glucose reading was $126 \mathrm{mg} / \mathrm{dL}$ or greater, or the participant took insulin or oral hypoglycemic drugs. Fasting total and high-density lipoprotein cholesterol and triglycerides were enzymatically determined, and low-density lipoprotein cholesterol was calculated according to Friedewald's formula. According to the Adult Treatment Panel III recommendations for patients with carotid atherosclerosis, ${ }^{27}$ patients with low-density lipoprotein cholesterol greater than $100 \mathrm{mg} / \mathrm{dL}$ or nonhigh-density lipoprotein cholesterol greater than $130 \mathrm{mg} / \mathrm{dL}$, or who were treated with lipid-lowering drugs, were considered hypercholesterolemic. The ankle-arm index was calculated by a senior physician according to carefully standardized procedures followed in our noninvasive vascular diagnostic laboratory. ${ }^{28-30}$

History of cerebrovascular accidents was investigated by a senior neurologist through direct interview and review of medical charts: transient ischemic attack (TIA) was defined as an abrupt onset of symptoms and/or signs related to a focal cerebral or visual deficit attributed to focal cerebral or retinal ischemia lasting less than 24 hours. ${ }^{31,32}$ Stroke was defined as an abrupt onset of symptoms and/or signs related to a focal and/or global deficit of cerebral functions lasting more than 24 hours and not attributable to causes other than cerebrovascular accident. ${ }^{32,33}$ Reversible ischemic neurologic disorder or minor stroke was defined as a stroke with no or minimal impairment in daily living independence (usually Rankin score $<3) .{ }^{31}$ According to the medical history of TIA, stroke, and minor stroke, patients were classified as symptomatic when they reported at least one cerebrovascular ischemic episode before $\mathrm{CE}$, and asymptomatic when they had no history of cerebrovascular symptoms. Only patients who had their first symptomatic ischemic episode in the year preceding CE were included. All patients underwent cerebral magnetic resonance (MR) imaging (MRI) evaluation before CE; presence and severity of cerebral ischemic lesions were evaluated by a qualified neuroradiologist. Infarct on MRI was defined as an area with abnormal signal intensity in a vascular distribution, without mass effect. ${ }^{34}$ Patients without history of cerebrovascular ischemic symptoms but with MRI evidence of infarction were excluded. Patients with clinical history of TIA but with MRI evidence of cerebral infarction were included among symptomatic patients. 
All patients underwent color Doppler ultrasonography evaluation of carotid arteries at the central vascular radiology laboratory of the hospital. Severity of stenosis was classified according to the North American Symptomatic Carotid Endarterectomy Trial criteria. ${ }^{35}$ A further confirmatory noninvasive imaging test (MR or computed tomographic angiography) was performed on selected patients on clinical judgement of the vascular surgeon.

All patients underwent a baseline evaluation of cognitive performance at the day hospital of our institute, following a standardized protocol based on clinical evaluation, including neurologic history and examination, and mental status examination. The cognitive assessment was performed through the age- and education-adjusted Mini Mental State Examination (MMSE) ${ }^{36}$ and the Clock Drawing Task (CDT). ${ }^{37}$ The MMSE ${ }^{36}$ primarily measures cognitive function in the dominant cerebral hemisphere, ${ }^{38}$ which is the left hemisphere in more than $98 \%$ of right-handed persons. ${ }^{39}$ MMSE has been shown to be a reliable instrument for cognitive screening in several settings, including the evaluation of cognitive impairment in patients with cerebrovascular disease. ${ }^{38,40,41}$ The range of MMSE score is from 0 to 30; higher scores indicate greater cognitive function. The $\mathrm{CDT}^{37}$ evaluates multiple areas of cognitive function including comprehension, memory, visuospatial abilities, abstract thinking, and executive function. ${ }^{37,42,43}$ Recently, some studies have referred the clock-drawing performances to "executive control" and suggest that CDT may have greater sensitivity than MMSE in detecting cerebrovascular disease mainly involving the frontal lobe. ${ }^{44,45}$ CDT has been shown to be a reliable screening tool for detecting cognitive impairment in older individuals. ${ }^{43,46-48}$ The CDT generally takes less than 5 minutes to administer and score. Individuals are verbally instructed to draw a clock, put all the numbers in, and set the time at 10 minutes after 11 . This time is reported to be the most sensitive for detecting neurocognitive dysfunction. ${ }^{49}$ Instructions (which are also written at the top of the page) may be repeated verbatim as needed. No cues are allowed; selfcorrection is permitted. Several scoring scales have been developed for the CDT as a screen for dementia. ${ }^{50-54}$ The score proposed by Sunderland et $\mathrm{al}^{37}$ was adopted in the current study: it is based on a set of criteria for evaluating clock drawings. The score range is from 1 to 10; higher score indicates better performance. Both MMSE and CDT were administered to each patient by the same qualified geriatrician who was blind to the history of cerebrovascular accidents. Baseline cognitive evaluation was performed within the month preceding CE in all patients. Patients with AD (diagnosed according to Diagnostic and Statistical Manual of Mental Disorders, Fourth Edition and National Institute of Neurologic Disorders and StrokeAlzheimer's Disease and Related Disorders Association [NINCDS-ADRDA] Work Group criteria), ${ }^{55,56} \mathrm{VaD}^{57}$ or with cognitive impairment (MMSE score $<25$ ) at baseline were excluded. The same cognitive evaluation, including MMSE and CDT, was repeated in all survivors at least 3 years after the surgical procedure, by the same qualified geriatrician.

\section{Statistical Analysis}

Statistical analysis was performed using software (SPSS Version 12.0 packaging for Windows, SPSS). A descriptive analysis of continuous and categorical variables was performed. The Chi square test was used to evaluate the association between categorical variables. The independent samples $t$ test procedure was used to evaluate differences in mean values between groups (it computes the differences between values of the two variables for each case and tests whether the average differs from 0 ). The paired samples $t$ test procedure was used to evaluate differences between two variables within group. The 1-way analysis of variance procedure for a quantitative variable by a single factor (independent) variable was used to test the hypothesis that several means are equal. Once determined the existence of differences among means, post hoc pairwise multiple comparisons (Bonferroni) were used to determine which means differed. Pairwise multiple comparisons test the difference between each pair of means and indicate significantly different group means at an alpha level of 0.05 .

\section{Results}

During the study period, there were 254 consecutive patients (age $\geq 65$ years) eligible for CE. A total of 57 patients were excluded (8 for evidence of cognitive impairment at baseline, 13 for MRI evidence of cerebral infarction without history of cerebrovascular ischemic episodes, and 36 because they resided outside the metropolitan area) and 65 refused to participate; 16 patients did not undergo surgical intervention. In all, 116 patients who underwent CE and agreed to participate in the study were enrolled and underwent baseline evaluation before CE procedure. A total of 13 patients died during the period of observation. The remaining 103 patients were considered for analysis. In all, 50 patients had LICA disease (29 symptomatic) and 53 patients had RICA disease (23 symptomatic). Among symptomatic patients, at least one TIA was reported by 32 patients $(61.5 \%)$, a minor stroke was ascertained in 10 patients (19.2\%), and a prior stroke was documented in 19 patients (36.5\%). Table 1 reports the main characteristics of the patients according to the side of carotid artery disease and the presence of ischemic symptoms; no significant differences were observed between groups. Table 2 shows the severity of ICA stenosis at baseline and at follow-up evaluation (36.4 \pm 4.5 months); bilateral stenosis was present in 6 asymptomatic and in 8 symptomatic patients. 
Table 1. Main characteristics of the patients at baseline

\begin{tabular}{|c|c|c|c|c|c|c|}
\hline & $\begin{array}{c}\text { LICA } \\
\text { symptomatic } \\
\mathrm{n}=29\end{array}$ & $\begin{array}{c}\text { LICA } \\
\text { asymptomatic } \\
n=21\end{array}$ & $\begin{array}{c}\text { RICA } \\
\text { symptomatic } \\
\mathrm{n}=23\end{array}$ & $\begin{array}{c}\text { RICA } \\
\text { asymptomatic } \\
n=30\end{array}$ & $\chi^{2}$ & $P$ \\
\hline Age, y (mean $\pm \mathrm{SD})$ & $72.9 \pm 6.7$ & $72.3 \pm 6.1$ & $72.4 \pm 7.2$ & $72.8 \pm 8.0$ & & \\
\hline Men/women & $21 / 8$ & $15 / 6$ & $17 / 6$ & $20 / 10$ & 0.078 & .779 \\
\hline Hypertension, $\mathrm{n}(\%)$ & $24(82.7)$ & $17(80.9)$ & $19(82.6)$ & $25(83.3)$ & 0.679 & .410 \\
\hline Hypercholesterolemia, n (\%) & $19(65.5)$ & $12(57.1)$ & $14(60.9)$ & $15(50.0)$ & 0.100 & .752 \\
\hline Diabetes, n (\%) & $11(37.9)$ & $7(33.3)$ & $6(26.1)$ & $10(33.3)$ & 0.442 & .342 \\
\hline Smokers, n (\%) & $20(69.0)$ & $17(80.9)$ & $17(73.9)$ & $22(73.3)$ & 0.152 & .697 \\
\hline CHD, n (\%) & $12(41.3)$ & $11(52.4)$ & $7(30.4)$ & $10(33.3)$ & 2.231 & .135 \\
\hline POAD, n $(\%)$ & $10(34.5)$ & $10(47.6)$ & $10(43.5)$ & $11(36.6)$ & 1.659 & .198 \\
\hline $\mathrm{AF}, \mathrm{n}(\%)$ & $2(6.9)$ & $2(9.5)$ & $2(8.7)$ & $2(6.6)$ & 0.115 & .734 \\
\hline
\end{tabular}

$A F$, Atrial fibrillation; $C H D$, coronary heart disease; LICA, left internal carotid study; POAD, peripheral obstructive arterial disease; RICA, right internal carotid artery.

In the overall sample, restenosis of the operated ICA occurred in 10 patients $(9.7 \%)$.

At the end of the study period, a diagnosis of dementia was made in 3 patients ( $2.9 \%$ of the overall sample). In the overall clinical evaluation, some cognitive decline during the period of observation (as detected by a significant reduction in MMSE or CDT score) occurred in 48 patients $(46.6 \%)$. Table 3 shows the MMSE and CDT scores at baseline and at the end of follow-up, according to the side of carotid artery disease and the presence of symptoms. The average time passed from CE to follow-up evaluation in the overall sample was $44.4 \pm 14.3$ months, without differences between groups. No significant differences were observed at baseline. At the end of

Table 2. Severity of internal carotid artery stenosis at baseline and at follow-up evaluation, determined by Doppler ultrasonography

\begin{tabular}{|c|c|c|c|c|}
\hline & $\begin{array}{l}\text { Asymptomatic } \\
\quad(\mathrm{n}=51)\end{array}$ & $\begin{array}{l}\text { Symptomatic } \\
\qquad(\mathrm{n}=52)\end{array}$ & & \\
\hline \multicolumn{5}{|c|}{ Baseline severity of stenosis: } \\
\hline \multicolumn{5}{|c|}{ ICA that underwent $\mathrm{CE}$} \\
\hline $50 \%-70 \%, \mathrm{n}(\%)$ & $0(0)$ & $4(7.7)$ & & \\
\hline $70 \%-90 \%, \mathrm{n}(\%)$ & $38(74.5)$ & $32(61.5)$ & & \\
\hline$>90 \%, \mathrm{n}(\%)$ & $13(25.5)$ & $16(30.8)$ & $t=4.815$ & $P=.090$ \\
\hline \multicolumn{5}{|l|}{ Controlateral ICA } \\
\hline$<50 \%$ & $26(51.1)$ & $36(69.2)$ & & \\
\hline $50 \%-70 \%, \mathrm{n}(\%)$ & $11(21.6)$ & $7(13.5)$ & & \\
\hline $70 \%-90 \%, \mathrm{n}(\%)$ & $11(21.6)$ & $4(7.7)$ & & \\
\hline$>90 \%, \mathrm{n}(\%)$ & $2(3.9)$ & $0(0)$ & & \\
\hline Occlusion & $1(2.0)$ & $5(9.6)$ & $t=10.490$ & $P=.062$ \\
\hline \multicolumn{5}{|l|}{ Follow-up evaluation: } \\
\hline \multicolumn{5}{|c|}{ ICA that underwent $\mathrm{CE}$} \\
\hline$<50 \%$ & $47(92.2)$ & $46(88.5)$ & & \\
\hline $50 \%-70 \%, \mathrm{n}(\%)$ & $1(2.0)$ & $1(2.0)$ & & \\
\hline $70 \%-90 \%, \mathrm{n}(\%)$ & $0(0)$ & $2(3.8)$ & & \\
\hline$>90 \%, \mathrm{n}(\%)$ & $2(3.9)$ & $3(5.8)$ & & \\
\hline Occlusion & $1(2.0)$ & $0(0)$ & $t=4.292$ & $P=.508$ \\
\hline \multicolumn{5}{|l|}{ Controlateral ICA } \\
\hline$<50 \%$ & $29(58.0)$ & $32(62.2)$ & & \\
\hline $50 \%-70 \%, \mathrm{n}(\%)$ & $10(20.0)$ & $9(17.6)$ & & \\
\hline $70 \%-90 \%, \mathrm{n}(\%)$ & $8(16.0)$ & $5(9.8)$ & & \\
\hline$>90 \%, \mathrm{n}(\%)$ & $1(2.0)$ & $0(0)$ & & \\
\hline Occlusion & $2(4.0)$ & $5(9.8)$ & $t=3.825$ & $P=.575$ \\
\hline
\end{tabular}

$C E$, Carotid endoarterectomy; ICA, internal carotid artery. 
Table 3. Mini Mental State Examination and Clock Drawing Task score at baseline and at the end of the study period, according to the side of carotid disease and the presence of symptoms

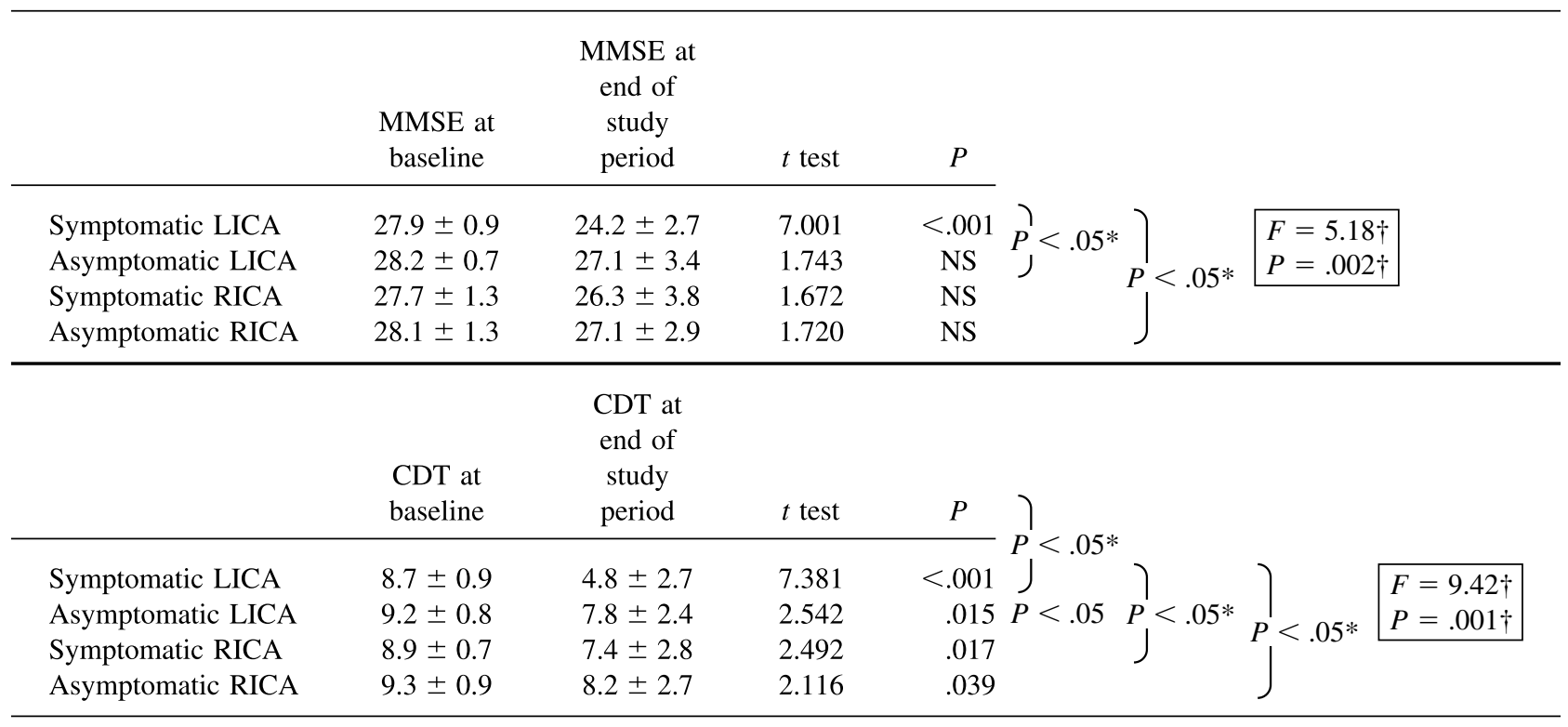

$C D T$, Clock drawing task; LICA, left internal carotid artery; MMSE, Mini Mental State Examination; NS, not significant; RICA, right internal carotid artery.

*Bonferroni test.

$\dagger$ One-way analysis of variance.

the study period, MMSE score was significantly reduced for patients with symptomatic LICA disease $(t=7.001, P$ $<$.001) but it was not for patients with asymptomatic LICA or for those with RICA disease. Overall, patients with symptomatic LICA disease had greater cognitive decline as measured by the MMSE score than other individuals $(\mathrm{F}=5.18, P=.002)$, most of this difference being accounted for by greater decline compared with patients with asymptomatic LICA and RICA $(P<.05$ both). At the end of the study period, a significant reduction in CDT score was observed in each group. Patients who underwent CE for symptomatic LICA disease had greater cognitive decline as measured by the CDT score than other individuals $(\mathrm{F}=9.42, P=.001)$, with significant differences between patients with symptomatic LICA and each other group of patients.

\section{Discussion}

The results of this study show that, in a period of 44 months, some cognitive decline occurred in roughly half of patients who underwent $\mathrm{CE}$, and dementia developed in $3 \%$ of these patients. Patients who received CE for symptomatic LICA disease had significantly greater risk of cognitive decline, determined either by the age- and education-adjusted MMSE or by the CDT, than patients operated on for asymptomatic LICA disease or for RICA disease, either symptomatic or asymptomatic. Silent ce rebral ischemia, and clinically evident cerebrovascular ischemic events, may be a cause of cognitive decline in older patients. $8,12,32,58,59$ The presence of high-grade stenosis of the ICA is associated with silent cerebral infarction and symptomatic infarction. ${ }^{60}$ Cerebrovascular ischemic episodes involving the left hemisphere, and asymptomatic high-grade stenosis of the LICA, are associated with increased risk of cognitive impairment. ${ }^{8-12,16}$ Although a direct relation between cerebrovascular ischemic symptoms and silent cerebrovascular ischemia has not been stated, it is reasonable to argue that patients with cerebrovascular ischemic symptoms may have a greater burden of cerebral ischemic than asymptomatic individuals and, therefore, a greater risk of cognitive decline. Our findings confirm this hypothesis and demonstrate that among patients undergoing $\mathrm{CE}$, those with symptomatic LICA stenosis have greater risk of cognitive decline than asymptomatic patients with LICA disease or patients with RICA disease, either symptomatic or asymptomatic. Therefore, our results suggest that patients with LICA disease might derive some additive benefit in the prevention of cognitive decline from endarterectomy before occurrence of cerebrovascular ischemic symptoms. However, specifically designed larger studies are needed to confirm these findings, before the threshold for endarterectomy or carotid stenting may change. 
Observational studies have suggested that cognitive function improves in some patients after endoarterectomy, but other studies show conflicting results. ${ }^{20-26}$ However, in most of these studies, differences between stenosis of the RICA and LICA, and between symptomatic and asymptomatic patients, were not evaluated. On this background, our findings indicate that nearly half of patients who undergo CE experience some cognitive decline, and that this risk is significantly increased in symptomatic patients with LICA disease. Previous studies demonstrated that, in a 5-year follow-up, 50\% of asymptomatic individuals with unoperated high-grade stenosis of the LICA had cognitive decline. ${ }^{16}$ Although a direct comparison between different studies is not feasible, our findings would suggest that CE might provide some protection against cognitive decline in these patients. Reduction in risk for cognitive decline might be an important additive benefit of surgical interventions to treat carotid artery disease. However, further study is warranted before considering CE in patients without history of cerebrovascular ischemic events.

Although both MMSE and CDT consistently detected a greater risk of cognitive decline in patients with symptomatic LICA than in other individuals, the CDT appeared more sensitive in revealing cognitive impairment among patients with cerebrovascular disease. The CDT has been used to evaluate mainly frontal functions and, particularly, visuospatial abilities in patients with AD. ${ }^{37,42,43}$ Recently, some studies referred the clockdrawing performances to executive control and suggested that CDT may have greater sensitivity than MMSE in detecting cerebrovascular disease mainly involving the frontal lobe. ${ }^{44,45}$ Although the predictive value of impaired performances in CDT has not been defined, our results show that CDT is extremely sensitive in recognizing cognitive impairment in patients who undergo operation for high-grade carotid stenosis. These findings add support to current evidence that CDT might be a useful tool for detecting an impairment in frontal executive functions, which is a hallmark of vascular cognitive impairment. ${ }^{61-63}$

Some limitations of this study should be addressed. In our view, the main limitation is probably represented by the relatively small sample, which may have underpowered our study to detect further significant differences between groups and may limit the generalization of results; current implementation of the sample size investigated may add further information. We decided to include only consecutive patients aged 65 years and older; this decision stems from the consideration that older patients are the most prevalent among candidates to $\mathrm{CE}$ and, at the same time, they have far greater risk of cognitive decline than younger patients. The symptomatic group was composed of patients with a varying clinical background; although a further distinction (e.g., stroke $v$ TIA) might provide a better opinion, this would have required a greater sample size to detect clinical differences. Moreover, this further distinction should not have affected the validity of current results, which suggest an increased incidence of cognitive decline in symptomatic patients with LICA disease. Finally, it might be argued that symptomatic patients have increased risk of cognitive decline compared with asymptomatic patients irrespective of whether patients received CE or not and that, therefore, the study would require a control group consisting of patients without operation. However, the inclusion of a control group with significant carotid disease, although asymptomatic, excluded from the surgical option, would not be ethically feasible. Moreover, it would not add to the question addressed in the current study, which is not whether CE is associated with cognitive decline, but whether CE performed before symptoms in patients with LICA disease may have potential benefit in the prevention of cognitive decline.

In conclusion, our results demonstrate that in a sample of older patients undergoing $\mathrm{CE}$, those who have symptomatic LICA disease have increased risk of cognitive decline. Therefore, it can be inferred that patients with LICA disease might derive some additive benefit in the prevention of cognitive decline from endarterectomy before occurrence of cerebrovascular ischemic symptoms. However, specifically designed larger studies are needed to confirm these findings, before the threshold for endarterectomy or carotid stenting may change.

\section{References}

1. Morris JC. The nosology of dementia. Neurol Clin 2000; 18:773-788.

2. Iadecola C, Gorelick PB. Converging pathogenic mechanisms in vascular and neurodegenerative dementia. Stroke 2003;34:335-337.

3. Ritchie K, Lovestone S. The dementias. Lancet 2002;360: 1759-1766.

4. Roman GC. Vascular dementia: Distinguishing characteristics, treatment and prevention. J Am Geriatr Soc 2003;5:S296-S304.

5. Snowdon DA, Greiner LH, Mortimer JA, et al. Brain infarction and the clinical expression of Alzheimer disease: The nun study. JAMA 1997;277:813-817.

6. Esiri MM, Nagy Z, Smith MZ, et al. Cerebrovascular disease and threshold for dementia in the early stages of Alzheimer's disease. Lancet 1999;354:919-920.

7. Zekry D, Duyckaerts R, Belmin J, et al. Degenerative and vascular lesions of the brain have synergistic effects in dementia of the elderly. Acta Neuropathol (Berl) 2002; 103:481-487.

8. Meyer JS, Rauch G, Rauch RA, et al. Risk factors for cerebral hypoperfusion, mild cognitive impairment, and dementia. Neurobiol Aging 2000;21:161-169.

9. Desmond DW, Moroney JT, Sano M, et al. Incidence of dementia after stroke. Stroke 2002;33:2254-2262.

10. Ballard C, Rowan E, Sthephens S, et al. Prospective follow-up study between 3 and 15 months after stroke: Improvements and decline in cognitive function among 
dementia-free stroke survivors $>75$ years of age. Stroke 2003;34:2440-2445.

11. Kokmen E, Whisnant JP, O'Fallon WM, et al. Dementia after ischemic stroke: A population-based study in Rochester, Minnesota (1960-1984). Neurology 1996;46:154-159.

12. Meyer JS, Quach M, Thornby J, et al. MRI identifies MCI subtypes: Vascular versus neurodegenerative. J Neurol Sci 2005;229:121-129.

13. Hachinski VC, Bowler JV. Vascular dementia. Neurology 1993;43:2159-2160.

14. O'Brien JT, Erkinjuntti T, Reisberg B, et al. Vascular cognitive impairment. Lancet Neurol 2003;2:89-98.

15. Rao R. The role of carotid stenosis in vascular cognitive impairment. Eur Neurol 2001;46:63-69.

16. Clairborne Johnston S, O'Meara ES, Manolio TA, et al. Cognitive impairment and decline are associated with carotid artery disease in patients without clinically evident cerebrovascular disease. Ann Intern Med 2004;140: 237-247.

17. Bakker FC, Kliyn CJM, Schinkel A, et al. Cognitive disorders in patients with occlusive disease of the carotid artery: A systematic review of the literature. J Neurol 2000;247:669-676.

18. Bakker FC, Kliyn CJM, Schinkel A, et al. Cognitive impairment in patients with carotid occlusion and ipsilateral transient ischemic attacks. J Neurol 2003;250:13401347.

19. Rothwell PM, Gutnikov SA, Fox AJ, et al, for the Carotid Endarterectomy Trailists' Collaboration. Analysis of pooled data from the randomized controlled trials of endarterectomy for symptomatic carotid stenosis. Lancet 2003;361:107-116.

20. Jacobs LA, Ganji S, Shirley JG, et al. Cognitive improvement after extracranial reconstruction for the low flowendangered brain. Surgery 1983;93:683-687.

21. Kelly MP, Garron DC, Javid H. Carotid artery disease, carotid endarterectomy and behaviour. Arch Neurol 1980;37:743-748.

22. Hemmingsen R, Mejsholm B, Boysen G, et al. Intellectual function in patients with transient ischemic attacks (TIA) or minor stroke: Long-term improvement after carotid andarterectomy. Acta Neurol Scand 1982;66:145-159.

23. Sirkka A, Salenius JP, Portin R, et al. Quality of life and cognitive performance after carotid endarterectomy during long-term follow-up. Acta Neurol Scand 1992;85:5862.

24. Matarazzo RG, Matarazzo JD, Gallo AE, et al. IQ and neuropsychological changes following carotid andarterectomy. J Clin Neuropsychol 1979;1:97-116.

25. Irvine CD, Gardner FV, Davies $\mathrm{AH}$, et al. Cognitive testing in patients undergoing carotid endarterectomy. Eur J Vasc Endovasc Surg 1998;15:195-204.

26. Lunn S, Crawley F, Harrison MJG, et al. Impact of carotid endarterectomy on cognitive functioning. Cerebrovasc Dis 1999;9:74-81.

27. Expert Panel on Detection, Evaluation, and Treatment of High Blood Cholesterol in Adults. Executive summary of the third report of the national cholesterol education program (NCEP) expert panel on detection, evaluation, and treatment of high blood cholesterol in adults (adult treatment panel III). JAMA 2001;285:2486-2497.

28. Zanocchi M, Bo M, Fonte G, et al. The ankle-arm blood pressure index is strongly predictive for cardiovascular mortality in men. Arch Gerontol Geriatr 1996;2223(suppl 5):157-166.

29. Fiandra U, Bo M, Poli L, et al. Correlation between ankle-arm blood pressure index (AAI) and atheroscle- rotic vascular involvement in coronary arteries. Arch Gerontol Geriatr 1996;22-23(suppl 5):85-94.

30. Bo M, Zanocchi M, Gallo R, et al. Prevalence and risk factors of peripheral arterial disease among elderly patients living in nursing homes. J Am Geriatr Soc 1996;44: 738-739.

31. WHO (World Health Organization). Cerebrovascular disorders: A clinical and research classification; Offset publication 1978; No 43. Geneva: World Health Organization, 1978.

32. Warlow C, Sudlow C, Dennis M, et al. Stroke. Lancet 2003;362:1211-1224.

33. Hatano S. Experience from a multicenter stroke register: A preliminary report. Bull World Health Organ 1976;54: 541-553.

34. Bryan RN, Manolio TA, Schertz LD, et al. A method for using MR to evaluate the effects of cardiovascular disease on the brain: The cardiovascular health study. AJNR Am J Neuroradiol 1994;15:1625-1633.

35. North American Symptomatic Carotid Endarterectomy Trial Collaborators. Beneficial effect of carotid endarterectomy in symptomatic patients with high-grade carotid stenosis. N Engl J Med 1991;325:445-453.

36. Folstein MF, Folstein SE, McHugh PR. Mini Mental State: A practical method for grading the cognitive state of patients for the clinician. J Psychiatr Res 1975;12:189-198.

37. Sunderland T, Hill JL, Mellow AM, et al. Clock drawing in Alzheimer's disease: A novel measure of dementia severity. J Am Geriatr Soc 1989;37:725-729.

38. Grace J, Nadler JD, White DA, et al. Folstein versus modified Mini-Mental State Examination in geriatric stroke: Stability, validity, and screening utility. Arch Neurol 1995;52:477-484.

39. Caplan D. Neurolinguistics and linguistic aphasiology an introduction. Cambridge: Cambridge University Press, 1987.

40. Dick JP, Guiloff RJ, Stewart A, et al. Mini-mental state examination in neurological patients. J Neurol Neurosurg Psychiatry 1984;47:496-499.

41. Nelson A, Fogel BS, Faust D. Bedside cognitive screening instruments: A critical assessment. J Nerv Ment Dis 1986;174:73-83.

42. Geldmacher DS, Whitehouse PJ. Evaluation of dementia. N Engl J Med 1996;335:330-336.

43. Shulman KI. Clock-drawing: Is it the ideal cognitive screening test? Int J Geriatr Psychiatry 2000;15:548-561.

44. Royall DR, Cordes JA, Polk MJ. CLOX: An executive clock drawing task. J Neurol Neurosurg Psychiatry 1998; 64:588-594.

45. Royall DR, Espino DV, Polk MJ, et al. Prevalence and patterns of executive impairment in community-dwelling Mexican Americans: Results from the Hispanic EPESE study. Int J Geriatr Psychiatry 2004;19:926-934.

46. Nishiwaki Y, Breeze E, Smeeth L, et al. Validity of the clock-drawing test as a screening tool for cognitive impairment in the elderly. Am J Epidemiol 2004;160:797807.

47. Death J, Douglas A, Kenny RA. Comparison of clockdrawing with mini-Mental State Examination as a screening test in elderly acute hospital admissions. Postgrad Med J 1993;69:696-700.

48. Juby A, Tench S, Baker V. The value of clock drawing in identifying executive dysfunction in people with a normal mini-Mental State Examination score. CMAJ 2002; 167:859-864. 
49. Freedman M, Kaplan E, Dells D. Clock drawing: A neuropsychological analysis. New York: Oxford University Press, 1994:3-8.

50. Wolf-Klein GP, Silverstone FA, Levy AP, et al. Screening for Alzheimer's disease by clock drawing. J Am Geriatr Soc 1989;37:730-734.

51. Rouleau I, Salmon DP, Butters N, et al. Quantitative and qualitative analyses of clock drawings in Alzheimer's and Huntington's disease. Brain Cogn 1992;18:70-87.

52. Sunderland T, Hill JL, Mellow AM, et al. Clock drawing in Alzheimer's disease: A novel measure of dementia severity. J Am Geriatr Soc 1989;37:725-729.

53. Mendez MF, Ala T, Underwood KL. Development of scoring criteria for the clock drawing task in Alzheimer's disease. J Am Geriatr Soc 1992;40:1095-1099.

54. Manos P. Ten-point clock test sensitivity for Alzheimer's disease in patients with MMSE score greater than 23. Int J Geriatr Psychiatry 1999;14:454-458.

55. Diagnostic and Statistical Manual of Mental Disorders. DSM-IV. 4th ed. Washington (DC): American Psychiatric Association, 1994.

56. McKahnn G, Drachman D, Folstein M. Clinical diagnosis of Alzheimer's disease: Reports of the NINCDS-ADRDA work group under auspices of Department of Health and Human Services task force on Alzheimer's disease. Neurology 1984;34:939-944.
57. Roman GC, Tatemichi TK, Erkinhutti T, et al. Vascular dementia: Diagnostic criteria for research studies; Report of the NINDS-AIREN international workshop. Neurology 1993;43:250-260.

58. Price TR, Manolio TA, Kronmal RA, et al. Silent brain infarction on magnetic resonance imaging and neurological abnormalities in community-dwelling older adults: The cardiovascular health study; CHS collaborative research group. Stroke 1997;28:1158-1164.

59. Longstreth WT Jr, Berick C, Manolio TA, et al. Lacunar infarcts defined by magnetic resonance imaging of 3660 elderly people: The cardiovascular health study. Arch Neurol 1998;55:1217-1225.

60. Norris JW, Zhu CZ. Silent stroke and carotid stenosis. Stroke 1992;23:483-485.

61. Roman GC, Royall DR. Executive control function: A rational basis for the diagnosis of vascular dementia. Alzheimer Dis Assoc Disord 1999:13(3):S69-S80.

62. Wolfe N, Linn R, Babikian VL, et al. Frontal systems impairment following multiple lacunar infarcts. Arch Neurol 1990;47:129-132.

63. Royall DR. Executive cognitive impairment: A novel perspective on dementia. Neuroepidemiology 2000;19: 293-299. 